\title{
BMJ Open Prescribing pattern of antipsychotic medication for first-episode psychosis: a retrospective cohort study
}

\author{
Dolores Keating (D) ,, ${ }^{1,2}$ Stephen McWilliams, ${ }^{3,4}$ Fiona Boland (iD , ${ }^{5}$ Roisin Doyle, ${ }^{6}$ \\ Caragh Behan, ${ }^{6}$ Judith Strawbridge, ${ }^{2}$ Mary Clarke ${ }^{4,6}$
}

To cite: Keating $D$, McWilliams S, Boland F, et al. Prescribing pattern of antipsychotic medication for first-episode psychosis: a retrospective cohort study. BMJ Open 2021;11:e040387. doi:10.1136/ bmjopen-2020-040387

- Prepublication history for this paper is available online. To view these files, please visit the journal online (http://dx.doi. org/10.1136/bmjopen-2020040387).

Received 12 May 2020

Revised 30 December 2020

Accepted 07 January 2021

Check for updates

(c) Author(s) (or their employer(s)) 2021. Re-use permitted under CC BY-NC. No commercial re-use. See rights and permissions. Published by BMJ.

${ }^{1}$ Pharmacy Department, Saint John of God Hospital, Dublin, Ireland

${ }^{2}$ School of Pharmacy, Royal College of Surgeons in Ireland, Dublin, Ireland

${ }^{3}$ Department of Medicine, Saint John of God Hospital, Dublin, Ireland

${ }^{4}$ School of Medicine and Medical Sciences, University College Dublin, Dublin, Ireland

${ }^{5}$ Data Science Centre and HRB Centre for Primary Care Research, Royal College of Surgeons in Ireland, Dublin, Ireland

${ }^{6}$ Dublin and East Treatment and Early Care Team, Blackrock, Co Dublin, Ireland

Correspondence to

Dolores Keating;

dolores.keating@sjog.ie

\section{ABSTRACT}

Objective Guidelines for antipsychotic use in first-episode psychosis (FEP) recommend that medication be chosen initially on the basis of side effect profile with doses at the lower end of the range. Our objective was to describe the pattern of antipsychotic use in FEP over a period of 21 years in the context of changing clinical guidelines and the development of specialist early intervention in psychosis (EIP) services.

Setting A community-based mental health service in South County Dublin (population 187 000) and a large private hospital.

Participants Participants included 465 patients with FEP (146 from an epidemiological study (1995-1999) and 319 from a specialist EIP service (2005-2016)). Treatment with antipsychotic medication did not exceed 30 days at study entry.

Outcome measures This is a descriptive study of prescribing practices in the context of service development and changing guidelines.

Results First-generation antipsychotics were prescribed for $65 \%$ of the early cohort compared with $4.3 \%$ of the EIP cohort. Olanzapine was initially prescribed for $79.7 \%$ of EIP patients. Initial doses of medication were frequently low ( $\leq 50 \%$ British National Formulary (BNF) maximum) in both cohorts $(71 \%$ and $78.6 \%)$. The demographic and clinical factors investigated did not influence the initial choice of antipsychotic medication significantly. Univariate logistic regression analysis suggested inpatient treatment setting was associated with a higher initial dose $(>50 \%$ BNF maximum) of antipsychotic medication. Increasing dose requirements over the first month of engagement with an EIP service was associated with poorer global functioning at baseline, greater positive symptoms at baseline and the inpatient treatment setting. However, these associations were not seen in the multivariable model.

Conclusions Second-generation antipsychotic prescribing predominates, but guidelines are often overlooked when choosing olanzapine notwithstanding lower initial dosages. EIP services should include proactive support for optimising medicines in line with evidence-based guidelines.

\section{INTRODUCTION}

Early intervention in psychosis (EIP) has been shown to reduce illness severity, reduce hospitalisation and improve aspects of social

\section{Strengths and limitations of this study}

- This 21-year study describes antipsychotic prescribing practices for a naturalistic cohort of patients with first-episode psychosis during two discrete periods before and after the introduction of an early intervention in psychosis service.

- All 465 patients had an objectively rated diagnosis of first-episode psychosis using validated instruments.

- All participants had little or no antipsychotic exposure before the study.

- A limitation of the study is its retrospective nature, meaning some data were missing.

- Rates of adherence to international prescribing guidelines may reflect the fact that they were not specifically promoted in this study setting.

functioning such as involvement in school or work. ${ }^{1}$ Benefits are sustained in the short term to medium term. ${ }^{23}$ The components of an EIP service differ with regard to the specific interventions offered. Common themes, however, include use of medication, psychosocial interventions such as cognitivebehavioural therapy, family interventions, rehabilitative interventions and psychoeducation. ${ }^{1}$ EIP models of care also vary with some services delivered by specialist standalone multidisciplinary teams and others by enhanced community mental health teams (CMHT) whereby staff within CMHTs care for people with EIP in addition to their usual roles. 'Hub and spoke' models involve a centralised specialist 'hub' which supports specialist staff or 'spokes' embedded in local CMHTs. ${ }^{4}$ Despite the variations in how the EIP services are delivered, recent evidence suggests that the early intervention approach is likely to be cost-effective. ${ }^{56}$

Antipsychotic medications are a key component of care for those experiencing psychosis. Response to a first antipsychotic medication in first-episode psychosis (FEP) is high with up to $80 \%$ achieving a reduction 
in symptoms. ${ }^{7}$ Maintenance treatment with antipsychotic medications reduces hospitalisations, improves life expectancy and enhances functional outcomes. ${ }^{8-11}$ Given the evidence that no one agent has shown significant superiority in terms of efficacy in this population, ${ }^{12}$ international guidelines recommend that tolerability should be the main influence when it comes to the choice of medication. ${ }^{13}$ Clozapine is generally reserved for those who have not adequately responded to antipsychotic treatment; however, lack of response should be identified early and clozapine initiated to improve outcomes. ${ }^{13} 14$ Furthermore, doses of medication should also be lower in FEP than those used to treat later episodes of schizophrenia because people experiencing FEP are particularly sensitive to the effects and side effects of antipsychotic medication.

Pharmacological treatment guidelines have evolved over the lifetime of early intervention services with a notable change being the role of second-generation antipsychotics (SGA).$^{15-17}$ The National Institute for Health and Care Excellence (NICE), for example, recommended SGAs as initial treatment in the early 2000s. Emerging evidence regarding the relative risks of SGAs, particularly metabolic risks, led to a change in the 2009 update of the NICE guidelines with initial choice being driven by side effect profile rather than classification of antipsychotics. ${ }^{17}$ The Patient Outcome Research Team (PORT) guidelines, also updated in 2009, specifically excluded olanzapine as a first-line treatment option ${ }^{16}$ and other guideline development groups have followed suit. ${ }^{15} 18$ EIP services vary in their approach to medication with limited published information on prescriber training, treatment goals, algorithms or guidelines and delivery of treatment. ${ }^{19}$ This is perhaps surprising given the evidence of suboptimal use of antipsychotic medication in clinical practice. ${ }^{2021}$

In this study, we describe the pattern of antipsychotic medication use in two cohorts of patients with FEP in the context of evolving clinical practice guidelines and the introduction of specialised EIP services. Our objectives were to determine (1) the adherence to international guideline recommendations on the initial choice and dose of antipsychotic medication, (2) whether a specific range of clinical or demographic factors at baseline were associated with the choice of medication or the initial dose of medication for patients supported by an EIP service.

\section{METHODS}

\section{Study design}

The study is a retrospective examination of the medication prescribed for two cohorts of patients with FEP before and after the introduction of EIP services. Data were gathered from clinical records, the EIP study database and electronic prescribing records. This article was written using the Strengthening the Reporting of Observational Studies in Epidemiology guidelines for reporting cohort studies. $^{22}$

\section{Study setting}

Data were extracted from a community-based mental health service located in an urban area of South County Dublin with a current population of approximately 187000. A large private hospital, located within the catchment area, also participated in the study. EIP services were preceded by an epidemiological First Episode Study (FES) between 1995 and $1999 .{ }^{23}$ Evidence from this study was used to secure funding for the Dublin and East Treatment and Early Care Team (DETECT). The specialist DETECT team offers rapid assessment leading to phasespecific psychological and family interventions. Antipsychotic medication use is managed by the patient's usual psychiatrist.

\section{Participants and inclusion criteria}

The FES cohort (C1) was an epidemiologically complete sample recruiting all patients presenting in the catchment area with a first lifetime episode of psychosis between 1995 and 1999. Patients were included if they were aged 12 or over, gave consent to participate and had received less than 30 days of antipsychotic treatment. Cases included in the DETECT cohort (C2) were assessed by the EIP service between 2005 and 2016 and gave consent to participate in the study. Participants were aged between 16 and 65 and had received fewer than 30 days of antipsychotic treatment before the EIP service assessment. At the time of assessment, informed consent was given by parents or guardians for all participants aged under 18 years in line with the study protocol and the requirements of the ethics committee. The cohorts are described in figure 1 .

\section{Assessments}

Participants were included if they had a diagnosis of FEP based on the Structured Clinical Interview for DSM-IV Axis I Disorders. ${ }^{24}$ The Global Assessment of Functioning (GAF) scale was used to rate subjectively social, occupational and psychological functions. Scores range from 100 (extremely high functioning) to 1 (severe impairment). ${ }^{25}$ For C1, psychological symptoms were assessed using the Positive and Negative Syndrome Scale (PANSS).$^{26}$ The PANSS is scored by summation of individual items to produce positive symptom and negative symptom domain scores in a range of 7-49 and a composite general psychopathology score in the range of 16-112. The Scale for the Assessment of Positive Symptoms (SAPS) and Scale for the Assessment of Negative Symptoms (SANS), wellestablished rating scales used in clinical research, were used to assess symptoms in C2. ${ }^{27}$ SANS measures negative symptoms on a 25-item, 6-point scale. Items are listed under the five domains of affective blunting, alogia, avolition/apathy, anhedonia/asociality and attention. SAPS measures positive symptoms on a 34-item, 6-point scale. Items are listed under hallucinations, delusions, bizarre behaviour and positive formal thought disorder. All scales were administered by trained clinicians who participated with inter-rater reliability. Duration of untreated psychosis (DUP) was defined as the interval between first 


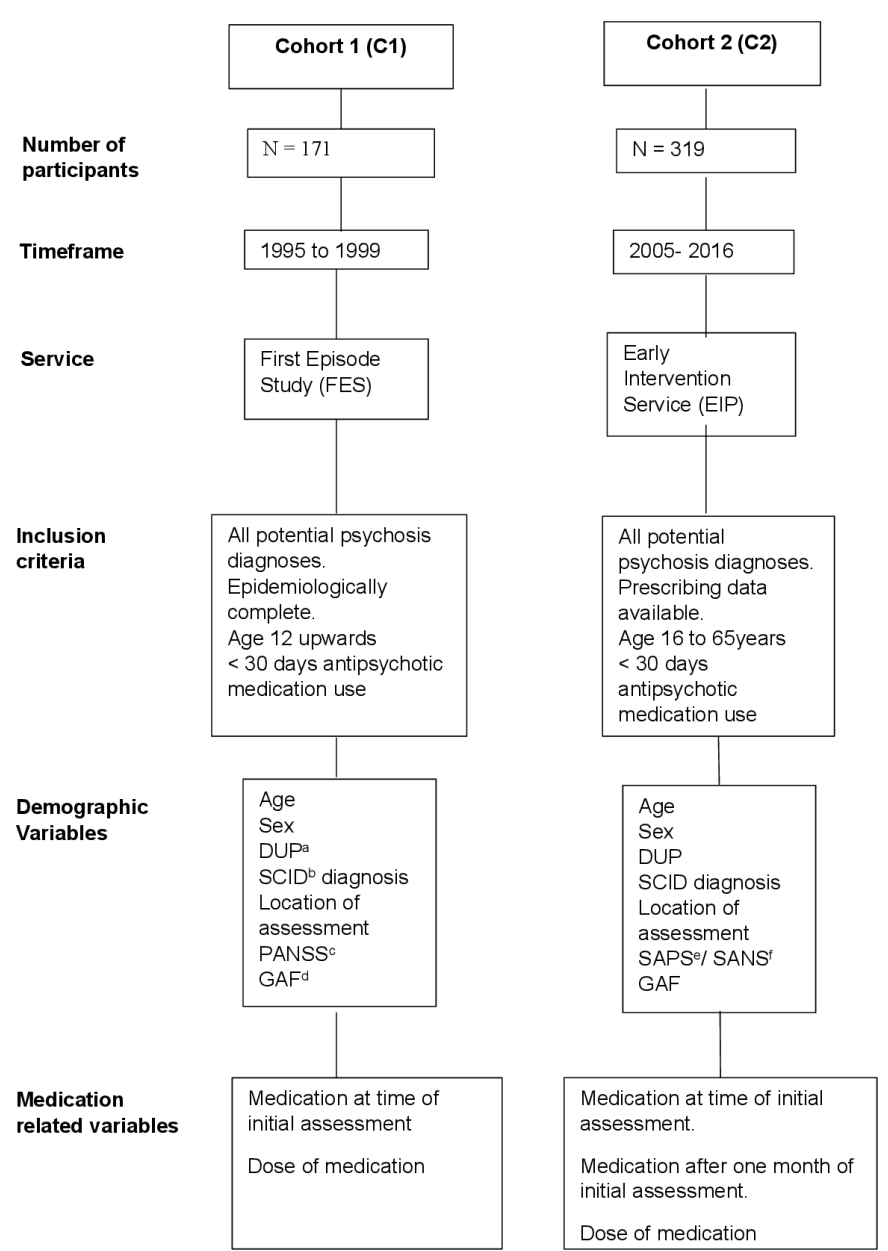

Figure 1 Description of cohorts of patients presenting to an early intervention service, time frame of presentation, inclusion criteria, demographic and medication-related variables. ${ }^{a}$ Duration of untreated psychosis. ${ }^{b}$ Structured Clinical Interview for DSM-IV Axis I Disorders. ' $P$ Positive and Negative Syndrome Scale. ${ }^{d}$ Global Assessment of Functioning. ${ }^{\text {e}}$ Scale for the Assessment of Positive Symptoms. 'Scale for the Assessment of Negative Symptoms. EIP, early intervention in psychosis.

experience of psychotic symptom(s) and presentation to the psychiatric services for initiation of treatment; first manic symptom(s) were used for bipolar disorder. ${ }^{28}$

\section{Antipsychotic prescribing data}

Prescribing data pertaining to $\mathrm{C} 1$ were compiled from paper charts. For the EIP cohort (C2), prescribing data at the time of clinical assessment (T1) were collected as part of a larger study of outcomes in FEP following the introduction of an EIP service. Medication at the time of initial assessment was recorded in the study database by the clinician carrying out the assessment. Data missing from the database and prescribing information following 1 month of engagement with the services (T2) were collected using hospital dispensing records and outpatient electronic prescribing records. Reports with details of prescription records were generated from the electronic health record separately using Discover Plus, a business intelligence software. It was taken that prescriptions generated within 1 week of the specified time points were the current medications. Cases for which no medication data were available were excluded.

Regular antipsychotic medications were included. Antipsychotics used for short periods on a 'pro re nata' basis or for rapid tranquilisation were excluded. Where medications were being switched, we considered this to be appropriate polypharmacy and included the new antipsychotic as the choice assuming that the switch would be completed.

Doses of antipsychotic medication were categorised into 'low', $<50 \%$ of the current British National Formulary (BNF) maximum dose; medium, $>51 \%$ to $<100 \%$ of current BNF maximum dose; and 'high' dose, $>100 \%$ of current BNF maximum dose. The rationale for this approach was based on pharmacological treatment guidelines which recommend doses at the lower end of the therapeutic dose range. ${ }^{13}$ An exception to this was risperidone for which $<6 \mathrm{mg}$ was categorised as a 'low' dose in FEP based on guideline recommendations. ${ }^{16}$ The current BNF dosing standards for haloperidol were applied but it should be noted that the BNF maximum dose has reduced over the lifetime of this study.

\section{Statistical methods}

Initially, descriptive statistics were used to describe baseline characteristics and general prescribing patterns in both cohorts. Means and SDs are reported for continuous variables and frequencies and percentages for categorical variables. For continuous scales which show evidence of or are expected to show some skew, a median and IQR is also presented. Scatterplots were used to display trends in olanzapine prescribing over time and an indicator included at 2009 when guidelines were first published advising against the use of olanzapine as an initial medication in FEP. Univariate and multivariable logistic regression analyses were used to explore potential demographic and clinical associations with olanzapine use (yes/no), dose initiated (medium/high vs low) and also change in dose (increased vs the same or decreased). Demographic and clinical variables included in the models were age, gender, DUP, GAF, SAPS, diagnosis and agitation symptoms. Statistical analysis was conducted using SPSS V.24 and Stata V.13.

\section{Patient and public involvement}

Patients and the public were not involved in this study.

\section{RESULTS}

\section{Demographic and clinical characteristics}

Demographic and clinical baseline data from the FES (C1) are described in table 1 and have previously been reported. ${ }^{28}$ This was an epidemiologically complete sample and all people presenting with FEP consented to participate. Demographic and clinical characteristics for the EIP service (C2) were included for those who consented to participate in the study and for whom prescribing data 
Table 1 Baseline description of demographic and clinical characteristics of two cohorts of patients presenting between 19951999 and 2005-2016 for assessment of first-episode psychosis prior to (C1) and after (C2) the introduction of an EIP service

\section{C1 (1995-1999) \\ $n=171$}

C2 (2005-2016) $\mathrm{n}=319$

\begin{tabular}{llr}
\hline Gender, $\mathrm{n}(\%)$ & & \\
Male & $99(58)$ & $189(59.2)$ \\
\hline Female & $72(42)$ & $130(40.8)$ \\
Age, mean (SD) & $28.5(11.1)$ & $32.5(11.3)$
\end{tabular}

\begin{tabular}{|c|c|c|c|c|}
\hline Inpatient on assessment, n (\%) & \multicolumn{3}{|c|}{$144(84.2)$} & $216(67.7)$ \\
\hline \multicolumn{5}{|l|}{ Initial diagnosis*, n (\%) } \\
\hline Schizophrenia spectrum & \multicolumn{3}{|c|}{$101(59.1)$} & $124(39.2)$ \\
\hline Substance-induced psychosis & \multicolumn{3}{|c|}{$12(7)$} & $45(14.2)$ \\
\hline Major depressive disorder & \multicolumn{3}{|c|}{$11(6.4)$} & $36(11.4)$ \\
\hline Bipolar disorder & \multicolumn{3}{|c|}{$25(14.6)$} & $35(11.1)$ \\
\hline Delusional disorder & \multicolumn{3}{|c|}{$13(7.6)$} & $35(11.1)$ \\
\hline Brief psychotic disorder & \multicolumn{3}{|c|}{0} & $22(7)$ \\
\hline \multirow[t]{2}{*}{ All other psychotic diagnoses } & \multicolumn{3}{|l|}{$4(5.2)$} & $19(6)$ \\
\hline & Mean & Median & Range & Median (IQR) \\
\hline DUP (months)† & 17.9 & 5 & $0.25-240$ & $3(0.63-13)$ \\
\hline GAF $\ddagger$ & \multicolumn{3}{|l|}{22.9} & $35(30-48.5)$ \\
\hline PANSS-Total & \multicolumn{4}{|l|}{74.4} \\
\hline PANSS-Negative & \multicolumn{4}{|l|}{15.7} \\
\hline PANSS-Positive & \multicolumn{4}{|l|}{21.3} \\
\hline \multicolumn{4}{|l|}{ SAPS-Total§ } & $18(10-31)$ \\
\hline \multicolumn{4}{|l|}{ SANS-Totalף } & $12(3-22)$ \\
\hline
\end{tabular}

*3 missing C2.

†5 missing C1; 156 missing C2.

\$6 missing $\mathrm{C} 2$.

$\$ 11$ missing $C 2$.

I14 missing C2.

DUP, estimated duration of untreated psychosis; EIP, early intervention in psychosis; GAF, Global Assessment of Functioning; PANSS-

Negative, Positive and Negative Syndrome Scale negative symptom score; PANSS-Positive, Positive and Negative Syndrome Scale positive symptom score; PANSS-Total, Positive and Negative Syndrome Scale total symptom score; SANS-Total, Scale for the Assessment of Negative Symptoms total score; SAPS-Total, Scale for the Assessment of Positive Symptoms total score.

were available (table 1). Participants in both time periods were predominantly male with an average age of 28.5 (SD 11.1) years in the early cohort and 32.5 (SD 11.3) years in the EIP cohort. For both cohorts, the majority were assessed in the inpatient setting and schizophrenia spectrum was the most common initial diagnosis (table 1).

\section{Choice of antipsychotic medication}

Prescribing data for a total of 465 patients were included, 146 in C1 and 319 in C2. Cases were excluded if prescribing data were not available for $\mathrm{C} 1 \quad(\mathrm{n}=25)$ or if there were no prescribing data at the time of initial assessment or 1-month follow-up for C2. Prescribing patterns of antipsychotic medications are described in table 2. The proportion of SGAs increased from $32.2 \%$ in $\mathrm{C} 1$ to over $90 \%$ in C2. First-generation antipsychotic (FGA) use predominated in $\mathrm{C} 1$ (65.1\%), of which the most frequently chosen was sulpiride $(19.2 \%)$, followed by thioridazine $(11 \%)$ and haloperidol (10.3\%). Olanzapine was the most frequently prescribed SGA throughout the time of the study and the prescribing frequency increased per year as represented in figure 2. Guidelines published in 2009 advising against the use of olanzapine as an initial medication in FEP and widening the choice to first or second-generation medicines did not appear to have an impact on prescribing patterns. Using C2 data, logistic regression analysis was used to explore demographic and clinical associations with olanzapine use (table 3). Univariate analysis showed evidence of an association with GAF scale, in that for every unit increase in GAF scale, the odds of being on olanzapine, compared with no olanzapine, 
Table 2 Antipsychotic prescribing patterns among two cohorts of patients presenting for assessment of first-episode psychosis before and after the introduction of an early intervention in psychosis service

\begin{tabular}{|c|c|c|c|}
\hline & \multirow{2}{*}{$\begin{array}{l}\text { Cohort } 1 \\
n=146 \\
\end{array}$} & \multicolumn{2}{|l|}{ Cohort 2} \\
\hline & & T1 $(n=305)$ & T2 $(n=293)$ \\
\hline & n (\%) & n (\%) & n (\%) \\
\hline \multicolumn{4}{|l|}{ Second generation } \\
\hline Olanzapine & $36(24.7)$ & $243(79.7)$ & $210(71.7)$ \\
\hline Risperidone (oral) & $8(5.5)$ & $25(8.2)$ & $22(7.5)$ \\
\hline Amisulpride & $2(1.4)$ & 7 (2.3) & $11(3.3)$ \\
\hline Quetiapine & $1(0.7)$ & $6(2.0)$ & $9(2.7)$ \\
\hline Aripiprazole & & $1(0.3)$ & $4(1.4)$ \\
\hline Risperidone LAI & & & $3(0.9)$ \\
\hline Paliperidone (oral) & & & $1(0.3)$ \\
\hline Paliperidone LAI & & & $5(1.5)$ \\
\hline Second generation total & $47(32.2)$ & $282(92.4)$ & $265(90.4)$ \\
\hline \multicolumn{4}{|l|}{ First generation } \\
\hline Sulpiride & $28(19.2)$ & $1(0.3)$ & $1(0.3)$ \\
\hline Thioridazine & $16(11)$ & $4(1.3)$ & \\
\hline Haloperidol & $15(10.3)$ & & $4(1.4)$ \\
\hline Chlorpromazine & $13(8.9)$ & $3(1)$ & $1(0.3)$ \\
\hline Trifluoperazine & $9(6.2)$ & $2(0.7)$ & $2(0.6)$ \\
\hline Flupenthixol depot & $4(2.7)$ & & $1(0.3)$ \\
\hline Pimozide & $4(2.7)$ & & $1(0.3)$ \\
\hline Zuclopenthixol depot & $1(0.7)$ & & $5(1.5)$ \\
\hline Zuclopenthixol oral & $1(0.7)$ & $1(0.3)$ & \\
\hline Flupenthixol (oral) & $1(0.7)$ & $2(0.7)$ & $1(0.4)$ \\
\hline Fluphenazine & $1(0.7)$ & & \\
\hline Pipotiazine & $1(0.7)$ & & \\
\hline Perphenazine & $1(0.7)$ & & \\
\hline First generation total & $95(65.1)$ & $13(4.3)$ & $16(5.5)$ \\
\hline No antipsychotic & $4(2.7)$ & $10(3.3)$ & $12(3.6)$ \\
\hline
\end{tabular}

LAI, long-acting injection; T1, time of initial assessment; T2, 1-month following initial assessment.

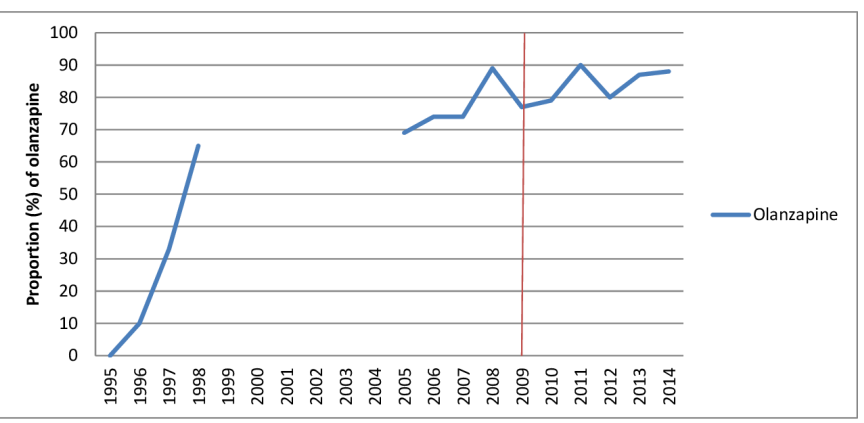

Figure 2 Proportion of olanzapine (\%) prescribed per year for patients presenting for assessment of first-episode psychosis. Guidelines published in 2009 advising against the use of olanzapine as an initial medication in first-episode psychosis (FEP) and widening the choice to first or secondgeneration antipsychotics (orange line). decreased (OR $0.97 ; 95 \%$ CI 0.95 to 0.99 ). However, there was no further evidence of associations with any other variables in univariate or multivariable analysis.

Data were available for C2 showing that $10(3.3 \%)$ patients at $\mathrm{T} 1$ and $11(3.9 \%)$ patients at $\mathrm{T} 2$ were not prescribed antipsychotic medications. At initial assessment, those who did not receive an antipsychotic medication had the following initial diagnoses: 'all other psychotic diagnosis' $(n=4)$, substance-induced psychosis, major depressive disorder $(n=2)$, brief psychotic episode $(n=2)$ and delusional disorder. However, these data were only identifiable for patients who received prescriptions for other medication on the electronic database and may be an underestimate.

Five patients were prescribed long-acting injection (LAI) or depot formulation of antipsychotic medication in C1. While no patient was initiated on an LAI at initial 
Table 3 Regression analysis describing the odds of olanzapine use with reference to clinical and demographic characteristics for patients presenting to an EIP service

\begin{tabular}{|c|c|c|c|c|c|}
\hline & \multicolumn{3}{|c|}{ Univariate analysis } & \multicolumn{2}{|c|}{ Multivariable analysis $(n=142)$} \\
\hline & $\mathbf{n}$ & OR (95\% Cl) & $P$ value & OR $(95 \% \mathrm{Cl})$ & $P$ value \\
\hline Age & 295 & 1.02 (0.99 to 1.05$)$ & 0.14 & 1.04 (1.00 to 1.08$)$ & 0.06 \\
\hline DUP (months) & 167 & 0.99 (0.97 to 1.01$)$ & 0.22 & 0.99 (0.97 to 1.01$)$ & 0.43 \\
\hline GAF & 291 & 0.97 (0.95 to 0.99$)$ & 0.02 & 0.98 (0.94 to 1.01$)$ & 0.18 \\
\hline SAPS & 289 & 1.06 (0.97 to 1.16$)$ & 0.18 & 1.00 (0.86 to 1.17$)$ & 0.97 \\
\hline Sex & 295 & & & & \\
\hline Male & & 1.00 & & 1.00 & \\
\hline Female & & 0.82 (0.45 to 1.51$)$ & 0.53 & 0.68 (0.28 to 1.63$)$ & 0.39 \\
\hline Treatment & 295 & & & & \\
\hline Outpatient & & 1.00 & & 1.00 & \\
\hline Inpatient & & 1.34 (0.72 to 2.51$)$ & 0.36 & 1.66 (0.66 to 4.19$)$ & 0.28 \\
\hline Diagnosis & 292 & & & & \\
\hline Affective & & 1.00 & & 1.00 & \\
\hline Schizophreniform & & 0.54 (0.25 to 1.18$)$ & 0.12 & 1.04 (0.36 to 3.05$)$ & 0.94 \\
\hline All other diagnoses & & 5.64 (0.69 to 46.39$)$ & 0.11 & 5.25 (0.53 to 52.08$)$ & 0.16 \\
\hline Agitation symptoms & 295 & & & & \\
\hline Present* & & 1.00 & & 1.00 & \\
\hline Not present $\dagger$ & & 1.25 (0.66 to 2.39$)$ & 0.50 & 0.86 (0.35 to 2.10$)$ & 0.74 \\
\hline
\end{tabular}

*Score of $2=$ mild, $3=$ moderate, $4=$ marked or $5=$ severe on the SAPS excitatory/agitation score.

†Score of $0=$ none or $1=$ questionable on the SAPS excitatory/agitation score.

DUP, estimated duration of untreated psychosis; EIP, early intervention in psychosis; GAF, Global Assessment of Functioning; SAPS, Scale for the Assessment of Positive Symptoms.

presentation for C2, $14(4.8 \%)$ had commenced an LAI by 1 month of treatment. Of the 319 cases in C2, data on both the medication used at initial assessment and at 1 month are available for 280 cases. Of these, 35 (12.5\%) patients required a switch of antipsychotic medication within 1 month. Risperidone $(\mathrm{n}=6,17.1 \%)$ was the most frequently used second-choice antipsychotic followed by amisulpride $(n=4,11.4 \%)$ and quetiapine $(n=3,8.6 \%)$.

\section{Dose of antipsychotic medication}

Doses of medication at initial assessment were generally low in both cohorts $(\mathrm{C} 1,71 \%$ and $\mathrm{C} 2,78.6 \%)$. In this study, logistic regression was used to explore potential demographic and clinical associations with the odds of medium/high dose, compared with low dose (table 4). Univariate analysis showed that the odds of medium/high dose, compared with low dose, was significantly higher for an inpatient compared with an outpatient (OR 2.36; 95\% CI 1.09 to 5.11). No further evidence of associations with any other variables in univariate or multivariable analysis was seen.

After 1 month of treatment the proportion of people in C2 requiring medium or high doses of medication increased from $17.9 \%$ to $42.7 \%$. Of these, $4(1.2 \%)$ patients were treated with doses above the BNF maximum, all of which were olanzapine at doses of $22.5-30 \mathrm{mg} /$ day.
Data on the dose of medication at both time points in C2 were available for 268 patients. Of these, 72 (26.8\%) required an increase in dose over the first month of engagement with the early intervention service (table 5). All of those who required an increase in dose had received an initial low dose of medication which was increased to a medium dose for 71 patients and a high dose for one patient. The dose of medication decreased for $10(3.7 \%)$ people between initial assessment and following 1 month of engagement with the service. All 10 had been started on a medium dose of antipsychotic and the dose was reduced to a low dose over the first month. Medication was discontinued for one person who initially started on a low dose of medication. The dose for 186 people $(69.4 \%)$ remained unchanged over the first month of engagement with the EIP service.

Univariate logistic regression analysis showed evidence that the odds of increasing a dose, compared with no increase (or a decrease), was significantly higher for an inpatient compared with an outpatient (OR 2.10; 95\% CI 1.09 to 4.05 , table 5). Additionally, there was evidence of associations with GAF and SAPS. For every unit increase in GAF scale, the odds of an increase, compared with no increase, decreased (OR 0.97; 95\% CI 0.94 to 0.99), and for every unit increase in SAPS the odds of an increase, compared with no increase, was 1.13 (95\% CI 1.05 to 
Table 4 Regression analysis exploring the odds of medium or high-dose antipsychotic use with reference to clinical and demographic characteristics for patients presenting to an EIP service

\begin{tabular}{|c|c|c|c|c|c|}
\hline & \multicolumn{3}{|c|}{ Univariate analysis } & \multicolumn{2}{|c|}{ Multivariable analysis $(n=142)$} \\
\hline & $\mathbf{n}$ & OR (95\% Cl) & $P$ value & OR (95\% Cl) & $P$ value \\
\hline Age & 280 & 0.98 (0.95 to 1.01$)$ & 0.11 & 0.97 (0.93 to 1.01$)$ & 0.18 \\
\hline DUP (months) & 154 & 0.97 (0.93 to 1.01$)$ & 0.09 & 0.98 (0.94 to 1.02$)$ & 0.23 \\
\hline GAF & 276 & 1.00 (0.98 to 1.02$)$ & 0.81 & 0.99 (0.95 to 1.03 ) & 0.50 \\
\hline SAPS & 274 & 1.05 (0.96 to 1.14$)$ & 0.28 & 0.97 (0.82 to 1.15$)$ & 0.73 \\
\hline Sex & 280 & & & & \\
\hline Male & & 1.00 & & 1.00 & \\
\hline Female & & 0.84 (0.45 to 1.57$)$ & 0.58 & 0.78 (0.28 to 2.14$)$ & 0.63 \\
\hline Treatment & 280 & & & & \\
\hline Outpatient & & 1.00 & & 1.00 & \\
\hline Inpatient & & 2.36 (1.09 to 5.11$)$ & 0.03 & 2.83 (0.79 to 10.15$)$ & 0.11 \\
\hline Diagnosis & 274 & & & & \\
\hline Affective & & 1.00 & & 1.00 & \\
\hline Schizophreniform & & 0.82 (0.39 to 1.73$)$ & 0.60 & 0.92 (0.29 to 2.98 ) & 0.89 \\
\hline All other diagnoses & & 1.83 (0.71 to 4.71 ) & 0.21 & 2.17 (0.4 to 11.89$)$ & 0.37 \\
\hline Agitation symptoms & 290 & & & & \\
\hline Present* & & 1.00 & & 1.00 & \\
\hline Not present† & & 0.94 (0.49 to 1.78$)$ & 0.83 & $0.75(0.26$ to 2.17$)$ & 0.59 \\
\hline
\end{tabular}

*Score of $2=$ mild, $3=$ moderate, $4=$ marked or $5=$ severe on the SAPS excitatory/agitation score.

†Score of $0=$ none or $1=$ questionable on the SAPS excitatory/agitation score.

DUP, estimated duration of untreated psychosis; EIP, early intervention in psychosis; GAF, Global Assessment of Functioning; SAPS, Scale for the Assessment of Positive Symptoms.

1.23). However, they did not remain significant in the multivariable analysis. There was no further evidence of associations with any other variables in univariate or multivariable analysis (table 5 ).

\section{DISCUSSION}

\section{Summary of findings}

This study describes the pattern of antipsychotic prescribing for a naturalistic cohort of patients presenting for assessment of FEP in a geographically defined catchment over a 21-year period. The data demonstrate the changes over time in the choice of antipsychotic medication, the move towards predominantly SGA use and the prevalence of olanzapine as a first-choice medication. Guidelines issued in both Europe and America widening the choice of antipsychotic medication or specifically not recommending olanzapine as an initial choice of agent do not appear to have had an impact on prescribing patterns. Additional indicators of good practice such as the use of low doses of antipsychotic medication for the initial treatment of FEP and the avoidance of high doses and antipsychotic polypharmacy are demonstrated. The demographic and clinical factors investigated did not appear to significantly influence the initial choice of antipsychotic medication. There was some evidence that inpatient treatment setting was associated with a higher initial dose of antipsychotic medication $(>50 \% \mathrm{BNF}$ maximum). Increasing dose requirements over the first month of engagement with an EIP service was associated with poorer global functioning at baseline, greater positive symptoms at baseline and the inpatient treatment setting. However, these associations were not seen in the multivariable model.

Comparison with previous literature and clinical implications EIP services aim to provide timely access to comprehensive assessment and programmes of care including medical, psychological, occupational and social support. ${ }^{29}$ A positive first experience of using antipsychotic medicines is likely to have an impact on future engagement with services and outcomes. ${ }^{30}$ Careful consideration of the first antipsychotic medication involves balancing side effects with expected benefits and incorporating the patient perspective through a shared decision-making approach. Managing side effects is a significant challenge with the risks of metabolic abnormalities, sexual problems and movement disorders among the many potential disadvantages of using these medications. Given the variety of antipsychotic medication available, the lack of evidence for relative efficacy benefits in FEP in the context of significant differences in side effect profiles, ${ }^{12}$ it is useful to examine what medications are actually used in practice with clinical implications for the services' 
Table 5 Regression analysis exploring the odds of an increase in dose (compared with no increase-stay the same or decreased) with reference to clinical and demographic characteristics for patients presenting to an EIP service

\begin{tabular}{|c|c|c|c|c|c|}
\hline & \multicolumn{3}{|c|}{ Univariate analysis } & \multicolumn{2}{|c|}{ Multivariable analysis $(n=142)$} \\
\hline & $\mathrm{n}$ & OR (95\% Cl) & $P$ value & OR $(95 \% \mathrm{Cl})$ & $P$ value \\
\hline Age & 268 & 0.98 (0.96 to 1.00$)$ & 0.11 & 0.99 (0.95 to 1.03$)$ & 0.51 \\
\hline DUP (months) & 147 & 0.99 (0.97 to 1.01$)$ & 0.37 & 1.00 (0.97 to 1.02$)$ & 0.76 \\
\hline GAF & 263 & 0.97 (0.94 to 0.99$)$ & $<0.01$ & 0.97 (0.93 to 1.01$)$ & 0.10 \\
\hline SAPS & 262 & $1.13(1.05$ to 1.23$)$ & $<0.01$ & 1.04 (0.89 to 1.21$)$ & 0.61 \\
\hline Sex & 268 & & & & \\
\hline Male & & 1.00 & & 1.00 & \\
\hline Female & & 0.8 (0.46 to 1.40$)$ & 0.44 & 1.37 (0.57 to 3.29$)$ & 0.48 \\
\hline Treatment & 268 & & & & \\
\hline Outpatient & & 1.00 & & 1.00 & \\
\hline Inpatient & & 2.10 (1.09 to 4.05$)$ & 0.03 & $1.76(0.61$ to 5.06$)$ & 0.30 \\
\hline Diagnosis & 265 & & & & \\
\hline Affective & & 1.00 & & 1.00 & \\
\hline Schizophreniform & & 0.83 (0.43 to 1.61$)$ & 0.58 & 0.68 (0.23 to 2.01$)$ & 0.48 \\
\hline All other diagnoses & & 1.13 (0.46 to 2.81$)$ & 0.79 & 1.43 (0.29 to 7.12$)$ & 0.67 \\
\hline Agitation symptoms & 263 & & & & \\
\hline Present* & & 1.00 & & 1.00 & \\
\hline Not present $†$ & & 1.66 (0.95 to 2.91$)$ & 0.07 & 1.23 (0.5 to 3.06$)$ & 0.65 \\
\hline
\end{tabular}

*Score of $2=$ mild, $3=$ moderate, $4=$ marked or $5=$ severe on the SAPS excitatory/agitation score.

†Score of $0=$ none or 1 =questionable on the SAPS excitatory/agitation score.

.DUP, estimated duration of untreated psychosis; EIP, early intervention in psychosis; GAF, Global Assessment of Functioning; SAPS, Scale for the Assessment of Positive Symptoms.

approach to managing physical health complications of antipsychotic use.

The trend towards SGA use over time in our study reflects the early optimism for medications with reduced propensity to cause anticholinergic side effects and long-term movement disorders. While the preference for olanzapine as a first-choice antipsychotic has been previously reported in the literature ${ }^{32-35}$ the prescribing rate in this cohort is high by comparison. For example, a Spanish study of prescribing practices for FEP found that $22.7 \%$ were prescribed olanzapine and a UK study described a prescribing rate of $35 \%$. In the USA, where the PORT guidelines specifically exclude olanzapine as a first-choice medication, the prescribing rate was $31.2 \%$ in the Recovery After an Initial Schizophrenia Episode-Early Treatment Programme (RAISE-ETP) study. ${ }^{20}$ Although this study did not explore the reasons for clinicians' choice of antipsychotic medication, olanzapine may be perceived to be more effective ${ }^{36}$ and reduce the need for additional prescribing, for example, a benzodiazepine or hypnotic.

Olanzapine has a higher risk of inducing weight gain and metabolic abnormalities in comparison to other antipsychotics that could potentially be used as an initial treatment option in FEP. ${ }^{3738}$ Antipsychotic-induced weight gain causes considerable patient distress, has serious general health implications and leads to early discontinuation of medication. ${ }^{39}$ Over time the characteristics of the population changed with more people provisionally diagnosed with substance use disorder in comparison to the early cohort. This likely reflects the achievements of the EIP service in reducing DUP and the diagnostic criteria for schizophrenia requiring presence of symptoms for 6 months or more. Olanzapine is a sedative medicine and may be a reasonable choice if the patient were agitated, a presentation commonly associated with substance misuse. However, univariate and multivariate regressions did not find an association with symptoms of agitation. While a reduction in the proportion of patients with FEP using olanzapine as an initial treatment could be beneficial, strategies to prevent and manage weight gain should form part of an EIP programme where olanzapine continues to be used as a first-line agent. Lifestyle interventions, ${ }^{40}$ metformin $^{41}$ and liraglutide ${ }^{42}$ are potential options.

Historically, LAI and depot formulations were recommended if adherence to oral medication was poor ${ }^{13}$ or, in more recent times, as first-line agents if the patient expressed a preference for the formulation. ${ }^{43}$ The advantages may include reduced hospitalisation, more stable therapeutic blood levels with no additional side effect burden and convenience for the patient. ${ }^{43}{ }^{44}$ Confirming adherence through the use of LAIs may lead to better treatment decisions and earlier recognition of treatment resistance. However, barriers to the use of these 
formulations include a reluctance on the part of some patients to engage in their use and a view that there may be a coercive nature to injecting medication. ${ }^{45}$ In this study, the prevalence of LAI use is low, with some historical use of the FGAs described in our first cohort. The preference for SGAs may have had an impact on the use of LAIs until the development of the first second-generation LAI formulation of risperidone.

Clozapine is generally reserved for patients whose symptoms have not responded to adequate trials of two antipsychotic medications at the maximum tolerable dose. ${ }^{13} 46$ When compared with chlorpromazine as an initial treatment for FEP, clozapine was no more effective. ${ }^{47}$ However, early use of clozapine for those considered treatment resistant has been recognised as increasingly important. For example, early use of clozapine was effective for $75 \%$ of those with treatment resistance included in an observational study by Agid et al. ${ }^{14}$ Furthermore, Yoshimura et al report that early use of clozapine was associated with a response rate of $80 \%$ compared with a response rate of $30 \%$ if clozapine initiation was delayed by 2.8 years or more. ${ }^{48}$ In our study, none of the patients were treated with clozapine and this is likely due to the inclusion of patients in the very early stages of treatment with up to 30 days of antipsychotic exposure at study entry. Additional research has demonstrated that the time to clozapine treatment for those with treatment-resistant illness in our study cohorts is reducing with an average time to clozapine treatment of 6.7 years in the FEP study ${ }^{49}$ compared with 2.1 years for those engaged in the EIP service. ${ }^{50}$

Guidelines recommend commencing antipsychotic medication at the lower half of the dose range in FEP. ${ }^{16} 17$ We therefore took a pragmatic approach to describing the pattern of antipsychotic doses by expressing dose as a percentage of the BNF maximum. Guideline recommendations were generally adhered to with $78.6 \%$ of patients prescribed lower doses at initial presentation and the use of high-dose medication regimens was negligible at both initial assessment and after 1 month of treatment. Bioque et al reported that $8.9 \%$ of patients received higher doses of medication, by comparison. ${ }^{34}$ Our description of antipsychotic use in the very early stages of treatment for FEP may explain the low rates of antipsychotic polypharmacy and high-dose treatment strategies in comparison to other studies. $^{3451}$

Clinical practice guidelines in psychiatry are often difficult to implement. ${ }^{21} 52$ In the RAISE-ETP study, for example, Robinson et alfound that, at the point of engagement with an EIP service, medication review would be beneficial for $39.4 \%$ of the 404 patients enrolled in their study. The reasons for medication review included the use of olanzapine $(31.2 \%)$ and the use of high-dose regimens $(8.8 \%)$ or combinations of antipsychotic medications $(23.3 \%) .{ }^{51}$ Proactive support for prescribing practice can be an effective means of improving the quality of medication use in FEP. ${ }^{19} 4853$ Observational studies by Yoshimura et al and Yeisen $e t$ aldemonstrated that the initial choice of antipsychotic can be influenced by locally implemented algorithms. ${ }^{48} 53$ Robinson et al developed the NAVIGATE prescribing principals and the COMPASS decisionmaking tool which was designed to facilitate communication between the patient and the prescriber in the RAISE trial. ${ }^{19}$ Training was provided for prescribers and they were given ongoing support throughout the study. Over a 2-year period, study participants $(n=223)$ had more medication visits, were more likely to use a medication that conformed to the NAVIGATE guidelines, experienced fewer side effects and gained less weight than those who had received usual community care $(n=181)$. Adherence estimator scores also improved in the NAVIGATE group but not in the community group. The models of care for EIP internationally give varying attention to supporting medicines optimisation. ${ }^{1}$ This evidence and the results of our study suggest that EIP services and patients could benefit from proactive support for prescribing practice.

\section{Strengths, limitations and future research}

We report prescribing data from a naturalistic cohort with inclusion criteria reflecting the age range and diagnoses presenting to an EIP service. The longitudinal data allow a view of the pattern of prescribing practice over a 21-year period during the development and implementation of an EIP service. We were also able to describe the clinical use of the medications in terms of dose changes and the need to switch medication or formulation over the first month of engagement with the EIP service. In studies regarding antipsychotic use in an FEP population, patients were often treated with antipsychotic medication for a number of months before assessment by an EIP service and therefore may not accurately reflect the first choice of antipsychotic or initial dose. ${ }^{32} 343551$ In our study, participants had less than 30 days of antipsychotic exposure.

Patient-related factors other than those assessed, such as patient preference sociodemographic factors or clinical metabolic parameters, may have had an influence on the choice or dose of antipsychotic medication. While we were able to describe the choice of antipsychotic when switching medication, we did not have the data to explore the reasons for switching medication. The retrospective nature of this study led to some missing data in both cohorts. The pattern of prescribing in the interim period between the FES and the EIP studies could not be described. International prescribing guidelines are not specifically promoted in Ireland and there are no local antipsychotic prescribing guidelines for FEP in the Irish mental health services. Their influence may, therefore, be expected to be poor. It would be useful to examine the topic prospectively to include shared decisionmaking processes and clinician-related factors and investigate the impact on patient outcomes including physical health. Future local or national guidelines may influence prescribing practice and include decision support tools and proactive management protocols to mitigate the potential side effects of antipsychotic medication. 


\section{CONCLUSION}

There is clearly a move towards the use of SGAs as initial treatment for FEP. Guidelines which recommend avoiding olanzapine as an initial choice based on its side effect profile do not appear to have had an influence on prescribing practice. Antipsychotics are generally initiated at low doses. Given the importance of early experiences with medication, consideration should be given to including a proactive approach to medicines optimisation within the EIP model of care. This could include locally agreed guidelines, decision support tools for both patients and clinicians and active management of side effects.

Acknowledgements The authors thank Ms Felicity Fanning and Mr Donal O'Keeffe for their contribution to this research. The authors also acknowledge the contribution of the numerous clinicians who collected the data over many years as part of the First Episode Study and the DETECT Early Intervention in Psychosis service.

Contributors DK designed and conducted the study in collaboration with JS and MC. DK extracted the data for the DETECT cohort from the DETECT research database and hospital prescribing records. $\mathrm{RD}$ and $\mathrm{CB}$ extracted the data in relation to the First Episode Study. FB and DK analysed the data. DK wrote the manuscript with input from SMcW, FB, JS and MC.

Funding The authors have not declared a specific grant for this research from any funding agency in the public, commercial or not-for-profit sectors.

Competing interests None declared.

Patient and public involvement Patients and/or the public were not involved in the design, or conduct, or reporting, or dissemination plans of this research.

Patient consent for publication Not required.

Ethics approval Ethics approval was granted by the Saint John of God Hospitaller Services Research Ethics Committee.

Provenance and peer review Not commissioned; externally peer reviewed.

Data availability statement Anonymised participant data are held in a secure research server and will be handled in accordance with the ethical approval for this project.

Open access This is an open access article distributed in accordance with the Creative Commons Attribution Non Commercial (CC BY-NC 4.0) license, which permits others to distribute, remix, adapt, build upon this work non-commercially, and license their derivative works on different terms, provided the original work is properly cited, appropriate credit is given, any changes made indicated, and the use is non-commercial. See: http://creativecommons.org/licenses/by-nc/4.0/.

\section{ORCID iDs}

Dolores Keating http://orcid.org/0000-0001-7507-2680

Fiona Boland http://orcid.org/0000-0003-3228-0046

\section{REFERENCES}

1 Correll CU, Galling B, Pawar A, et al. Comparison of early intervention services vs treatment as usual for early-phase psychosis: a systematic review, meta-analysis, and meta-regression. JAMA Psychiatry 2018;75:555-65.

2 Albert N, Melau M, Jensen $\mathrm{H}$, et al. Five years of specialised early intervention versus two years of specialised early intervention followed by three years of standard treatment for patients with a first episode psychosis: randomised, superiority, parallel group trial in Denmark (OPUS II). BMJ 2017;356:i6681.

3 Craig TKJ, Garety P, Power P, et al. The Lambeth early onset (Leo) team: randomised controlled trial of the effectiveness of specialised care for early psychosis. BMJ 2004;329:1067.

4 Behan C, Masterson S, Clarke M. Systematic review of the evidence for service models delivering early intervention in psychosis outside the stand-alone centre. Early Interv Psychiatry 2017;11:3-13.

5 Aceituno D, Vera N, Prina AM, et al. Cost-Effectiveness of early intervention in psychosis: systematic review. $\mathrm{Br} J$ Psychiatry 2019;215:388-94.
6 Behan C, Cullinan J, Kennelly B, et al. Estimating the cost and effect of early intervention on in-patient admission in first episode psychosis. J Ment Health Policy Econ 2015;18:57-62.

7 Zhu Y, Li C, Huhn M, et al. How well do patients with a first episode of schizophrenia respond to antipsychotics: a systematic review and meta-analysis. Eur Neuropsychopharmacol 2017;27:835-44.

8 Zhang C, Chen M-J, Wu G-J, et al. Effectiveness of Antipsychotic Drugs for 24-Month Maintenance Treatment in First-Episode Schizophrenia: Evidence From a Community-Based "Real-World" Study. J Clin Psychiatry 2016;77:e1460-6.

9 Lally J, Ajnakina O, Stubbs B, et al. Remission and recovery from first-episode psychosis in adults: systematic review and meta-analysis of long-term outcome studies. Br J Psychiatry 2017;211:350-8.

10 Hui CLM, Honer WG, Lee EHM, et al. Long-Term effects of discontinuation from antipsychotic maintenance following first-episode schizophrenia and related disorders: a 10 year follow-up of a randomised, double-blind trial. Lancet Psychiatry 2018;5:432-42.

11 Tiihonen J, Wahlbeck K, Lönnqvist J, et al. Effectiveness of antipsychotic treatments in a nationwide cohort of patients in community care after first hospitalisation due to schizophrenia and schizoaffective disorder: observational follow-up study. BMJ 2006;333:224.

12 Zhu Y, Krause M, Huhn M, et al. Antipsychotic drugs for the acute treatment of patients with a first episode of schizophrenia: a systematic review with pairwise and network meta-analyses. Lancet Psychiatry 2017:4:694-705.

13 Keating D, McWilliams S, Schneider I, et al. Pharmacological guidelines for schizophrenia: a systematic review and comparison of recommendations for the first episode. BMJ Open 2017;7:e013881.

14 Agid O, Remington G, Kapur S, et al. Early use of clozapine for poorly responding first-episode psychosis. J Clin Psychopharmacol 2007;27:369-73.

15 Galletly C, Castle D, Dark F, et al. Royal Australian and New Zealand College of Psychiatrists clinical practice guidelines for the management of schizophrenia and related disorders. Aust N Z J Psychiatry 2016;50:410-72.

16 Buchanan RW, Kreyenbuhl J, Kelly DL, et al. The 2009 schizophrenia Port psychopharmacological treatment recommendations and summary statements. Schizophr Bull 2010;36:71-93.

17 National Institute for Health and Care Excellence. Psychosis and schizophrenia in adults: treatment and management. CG178, 2014. Available: https://www.nice.org.uk [Accessed 18 Aug 2015].

18 Osser DN, Roudsari MJ, Manschreck T. The psychopharmacology algorithm project at the Harvard South shore program: an update on schizophrenia. Harv Rev Psychiatry 2013;21:18-40.

19 Robinson DG, Schooler NR, Correll CU, et al. Psychopharmacological treatment in the RAISE-ETP study: outcomes of a manual and computer decision support system based intervention. Am J Psychiatry 2018;175:169-79.

20 Medication Prescription Practices for the Treatment of First Episode Schizophrenia-Spectrum Disorders. Data from the National Raise-ETP study. NEUROPSYCHOPHARMACOLOGY. NATURE PUBLISHING GROUP MACMILLAN BUILDING, 4 CRINAN ST, LONDON N1 9XW, ENGLAND, 2014.

21 Howes OD, Vergunst F, Gee S, et al. Adherence to treatment guidelines in clinical practice: study of antipsychotic treatment prior to clozapine initiation. Br J Psychiatry 2012;201:481-5.

22 von Elm E, Altman DG, Egger M, et al. The strengthening the reporting of observational studies in epidemiology (STROBE) statement: guidelines for reporting observational studies. PLoS Med 2007;4:e296.

23 O'Keeffe D, Hannigan A, Doyle R, et al. The iHOPE-20 study: Relationships between and prospective predictors of remission, clinical recovery, personal recovery and resilience 20 years on from a first episode psychosis. Aust N Z J Psychiatry 2019;53:10801092:1080-92.

24 First M, Spitzer R, Gibbon M. Structured clinical interview for DSMIV axis I disorders (SCID). New York: New York State Psychiatric Institute. Biometrics Research, 1995.

25 American Psychiatric Association. Diagnostic and statistical manual of mental disorders; revised. Washington DG: DSM-III-R, 1987.

26 Kay SR, Fiszbein A, Opler LA. The positive and negative syndrome scale (PANSS) for schizophrenia. Schizophr Bull 1987;13:261-76.

27 Andreasen NC. Scale for the Assessment of Positive Symptons:(SAPS). University of lowa, 1984.

28 Clarke M, Whitty P, Browne S, et al. Untreated illness and outcome of psychosis. Br J Psychiatry 2006;189:235-40.

29 Health Services Executice. Early intervention for psychosis model of care. Dublin, 2019. Available: https://www.hse.ie/eng/about/who/ 
cspd/ncps/mental-health/psychosis/resources/ [Accessed 07 Jul 2019].

30 Lambert M, Conus P, Eide P, et al. Impact of present and past antipsychotic side effects on attitude toward typical antipsychotic treatment and adherence. Eur Psychiatry 2004;19:415-22.

31 Lincoln TM, Jung E, Wiesjahn M, et al. The impact of negative treatment experiences on persistent refusal of antipsychotics. Compr Psychiatry 2016;70:165-73.

32 Whale R, Harris M, Kavanagh G, et al. Effectiveness of antipsychotics used in first-episode psychosis: a naturalistic cohort study. BJPsych Open 2016;2:323-9.

33 Clarke A-M, McLaughlin P, Staunton J. Retrospective study of first episode psychosis in the Dublin Southwest mental health service: demographics, clinical profile and service evaluation of treatment. Irish Journal of Psychological Medicine 2017:1-10.

34 Bioque M, Llerena A, Cabrera B, et al. A pharmacovigilance study in first episode of psychosis: psychopharmacological interventions and safety profiles in the PEPs project. Int $J$ Neuropsychopharmacol 2016;19:pyv121.

35 Tungaraza TE, Ahmed W, Chira C, et al. Prescribing pattern of clozapine and other antipsychotics for patients with first-episode psychosis: a cross-sectional survey of early intervention teams. Ther Adv Psychopharmacol 2017;7:103-11.

36 Citrome L, McEvoy JP, Todtenkopf MS, et al. A commentary on the efficacy of olanzapine for the treatment of schizophrenia: the past, present, and future. Neuropsychiatr Dis Treat 2019;15:2559-69.

37 Tek C, Kucukgoncu S, Guloksuz S, et al. Antipsychotic-Induced weight gain in first-episode psychosis patients: a meta-analysis of differential effects of antipsychotic medications. Early Interv Psychiatry 2016;10:193-202.

38 De Hert M, Schreurs V, Sweers K, et al. Typical and atypical antipsychotics differentially affect long-term incidence rates of the metabolic syndrome in first-episode patients with schizophrenia: a retrospective chart review. Schizophr Res 2008;101:295-303.

39 Mustafa S, Joober R, Lepage M, et al. Predictors of 'all-cause discontinuation' of initial oral antipsychotic medication in first episode psychosis. Schizophr Res 2018;201:287-93.

40 Bonfioli E, Berti L, Goss C, et al. Health promotion lifestyle interventions for weight management in psychosis: a systematic review and metaanalysis of randomised controlled trials. BMC Psychiatry 2012;12:78.
41 de Silva VA, Suraweera C, Ratnatunga SS, et al. Metformin in prevention and treatment of antipsychotic induced weight gain: a systematic review and meta-analysis. BMC Psychiatry 2016;16:341.

42 Larsen JR, Vedtofte L, Jakobsen MSL, et al. Effect of liraglutide treatment on prediabetes and overweight or obesity in Clozapine- or Olanzapine-Treated patients with schizophrenia spectrum disorder: a randomized clinical trial. JAMA Psychiatry 2017;74:719-28.

43 Keepers GA, Fochtmann LJ, Anzia JM, et al. The American psychiatric association practice guideline for the treatment of patients with schizophrenia. Am J Psychiatry 2020;177:868-72.

44 Salgueiro M, Segarra R. Long-Acting injectable second-generation antipsychotics in first-episode psychosis: a narrative review. Int Clin Psychopharmacol 2019;34:51-6.

45 Parellada E, Bioque M. Barriers to the use of long-acting injectable antipsychotics in the management of schizophrenia. CNS Drugs 2016;30:689-701.

46 Barnes TR, Drake R, Paton C, et al. Evidence-Based guidelines for the pharmacological treatment of schizophrenia: updated recommendations from the British association for psychopharmacology. J Psychopharmacol 2020;34:3-78.

47 Lieberman JA, Phillips M, Gu H, et al. Atypical and conventional antipsychotic drugs in treatment-naive first-episode schizophrenia: a 52-week randomized trial of clozapine vs chlorpromazine. Neuropsychopharmacology 2003;28:995-1003.

48 Yoshimura B, Sato K, Takaki M, et al. Algorithm-based pharmacotherapy for first-episode schizophrenia involuntarily hospitalized: a retrospective analysis of real-world practice. Early Interv Psychiatry 2019;13:39-46.

49 Doyle R, Behan C, O'Keeffe D, et al. Clozapine use in a cohort of First-Episode psychosis. J Clin Psychopharmacol 2017;37:512-7.

50 Rowntree R, Murray S, Fanning F, et al. Clozapine use - has practice changed? J Psychopharmacol 2020;34:567-73.

51 Robinson DG, Schooler NR, John M, et al. Prescription practices in the treatment of first-episode schizophrenia spectrum disorders: data from the National RAISE-ETP study. Am J Psychiatry 2015;172:237-48.

52 Girlanda F, Fiedler I, Becker T, et al. The evidence-practice gap in specialist mental healthcare: systematic review and meta-analysis of guideline implementation studies. Br J Psychiatry 2017;210:24-30.

53 Yeisen RAH, Joa I, Johannessen JO, et al. Use of medication algorithms in first episode psychosis: a naturalistic observational study. Early Interv Psychiatry 2016;10:503-10. 